\title{
Notas taxonómicas sobre Pteridófitos chilenos
}

\section{Taxonomic notes on Chilean Pteridophyts}

\author{
Roberto Rodríguez Ríos \\ Departamento de Botánica, Facultad de Ciencias Naturales y Oceanográficas, Universidad de Concepción, Casilla \\ 160-C, Concepción, Chile. \\ rrodrigu@udec.cl

\section{RESUMEN}

En este trabajo se aclaran y rectifican varias confusiones taxonómicas en Pteridophyta chilenos: se mantiene el estatus de Adiantum sulphureum con su especie típica y con la variedad majus; Aspleniun fragile var. lomense se establece como nuevo sinónimo de Asplenium peruvianum; se establecen las diferencias entre Blechnum mochaenum y B. blechnoides y entre B. chilense y B. cordatum; se revalida Equisetum pyramidale; se considera a Polypodium masafuerae como especie endémica de Alejandro Selkirk (Archipiélago de Juan Fernández) y a P. pycnocarpum como una especie diferente de P. masafuerae, con una distribución en Perú, Bolivia, Argentina y en Chile sólo en la Región de Antofagasta.

Palabras clave: Pteridophyta chilenos, taxonomía, Adiantum, Asplenium, Blechnum, Equisetum, Polypodium.

\begin{abstract}
In this work many taxonomics mistakes for chilean Pteridophyta are clarify and corrected: Adiantum sulphureum with the tipical species maintain his status and the variety majus is revalided; Asplenium fragile var. lomense is a synonym of Asplenium peruvianum; the differences between Blechnum mochaenum and B. blechnoides and also B. chilense and B. cordatum are cleared; Equisetum pyramidale is revalidated; Polypodium masafuerae is a endemic species from Alejandro Selkirk (Juan Fernandez Archipelago), different of P. pycnocarpum from Peru, Bolivia, Argentina and Chile only in the Antofagasta Region.
\end{abstract}

KeYwords: Chilean Pteridophyta, taxonomy, Adiantum, Asplenium, Blechnum, Equisetum, Polypodium.

\section{INTRODUCCIÓN}

Al revisar en forma detenida las colecciones científicas de los pteridófitos chilenos y toda la información disponible en las obras clásicas y actuales sobre la validez de los nombres de los taxones que se encuentran en Chile, se ha evidenciado una identificación errónea de algunas especies, que ha llevado a confusiones tanto en el aspecto fitogeográfico como en la correcta aplicación de su nomenclatura.

En diversas circunstancias la identificación errónea de un material lleva a la descripción de nuevas especies, subespecies o variedades, sobre la base de un solo ejemplar a veces recolectado en regiones remotas de su distribución geográfica natural. Con el fin de aclarar algunos casos detectados en la flora pteridofítica de Chile, se analiza la nomenclatura y morfología de Adiantum sulphureum Kaulf., Asplenium fragile C.Presl var. lomense Weath., Blechnum blechnoides Keyserl., Blechnum chilense (Kaulf.) Mett., Botrychium australe R.Br. subsp. negeri (Christ) R.T.Clausen, Equisetum giganteum L. y Polypodium masafuerae Phil.

\section{MATERIALES Y MÉTODOS}

Se revisó la literatura pertinente para cada una de las especies en estudio y los materiales de los herbarios de CONC, GH, SGO (abreviaciones de acuerdo a Holmgren et al. 1990), que en el texto se mencionan como los más representativos dentro de su distribución geográfica, también se recurrió a la página www.plants.jstor.org para la obtención de información de los materiales tipos.

\section{RESULTADOS Y DISCUSIÓN}

\section{Adiantum SUlPHUREUm KaUlF.}

La primera descripción de Adiantum sulphureum pertenece a Kaulfuss (1824) "A. frondibus tripinnatis, pinnulis orbicularitrapeziis, subtus sulphureo-farinosis", sobre la base de un ejemplar recolectado por C. Chamisso en Talcahuano, Chile. La variedad típica de este helecho se encuentra 
desde la provincia de Colchagua hasta la de Capitán Prat, concentrándose en las regiones del Bío-Bío y de la Araucanía. Posteriormente, Hooker (1858) distingue dos variedades de Adiantum sulphureum, que poseen sustancias amarillas en la cara inferior de las pinnulas: a) var. minus "fronds four or five inches long, pinnules small, sori reniform" (que corresponde al typus de Kaulfuss) y b) var. majus "one foot high, pinnules larger, sori oblong reniform", que es de mayor tamaño y con los últimos segmentos casi totalmente cubiertos de sustancias amarillas.

Si se considera en conjunto la distribución de las dos variedades, esto abarcaría desde la provincia de Limarí hasta la de Capitán Prat (Rodríguez 1995).

J. Remy (1854) en la obra de C. Gay incluye a Adiantum sulphureum atribuido a Kaulfuss pero que de acuerdo a la descripción y a la foto del ejemplar recolectado en Colchagua por Claudio Gay en 1833, corresponde a Adiantum sulphureum var. majus Hook., además describe una nueva especie: Adiantum subsulphureum “... pínulas glabras por encima, cargadas por debajo de granulillos amarillos más o menos acercados, pero que no los cubren de una capa continua como en el $A$. sulphureum ... Especie más chiquita en todas sus partes que la precedente, habita las provincias centrales de la república". De acuerdo a esta descripción el taxón corresponde al Adiantum sulphureum Kaulf.

C. Meigen (1893) en su estudio de la vegetación de Santiago de Chile hace referencia a Adiantum sulphureum Kaulf. var. cuneifolium, sobre la base de un material recolectado en la Quebrada San Ramón: "Von der Hauptart, sowie auch von $A$. subsulphureum Remy sehr deutlich unterschieden durch keilförmige Blättchen". No he visto el pliego de Meigen, sin embargo, en los herbarios de CONC y SGO existen colecciones de la misma localidad y por la forma de los últimos segmentos descritos, coinciden con lo que Hooker llamó Adiantum sulphureum var. majus.

Finalmente, Giudice (1999) en su estudio de las especies argentinas del género Adiantum, trata a nuestra especie en cuestión bajo Adiantum chilense Kaulf. var. sulphureum (Hook.) Giudice, lo que es poco acertado porque Adiantum chilense tiene los últimos segmentos perfectamente glabros, coriáceos y de color verde oscuro, en cambio Adiantum sulphureum presenta los últimos segmentos subcoriáceos a membranáceos, de color verde claro y con gránulos harinosos de color amarillo en la superficie inferior.

Consecuente con lo anterior, se propone mantener el status de Adiantum sulphureum con su especie típica y una variedad propuesta por Hooker (1858), que se pueden separar mediante la siguiente clave:
Adiantum sulphureum Kaulf. var. sulphureum, Enum. Filic. 207. 1824. "Habitat in Chili, Chamisso". (Typus PH ! N 00000024).

Adiantum sulphureum Kaulf. var. minus Hook., Sp. Filic. 2: 44, tab. 76 A, figs. 1 y 2. 1858.

Adiantum subsulphureum Remy in Gay, Fl. Chile 6: 486. 1854. “province de Colchagua, Chili, Gay 1833”. (Phototypus P !). Adiantum aethiopicum L. var. sulphureum (Kaulf.) Kuntze, Revis. Gen. Pl. 3(2,2): 376. 1898.

Adiantum chilense Kaulf. var. sulphureum (Kaulf.) Kuntze ex Hicken, Revista Mus. La Plata 15: 261. 1908.

Adiantum sulphureum Kaulf. var. subsulphureum (Remy) Looser, Physis (Buenos Aires) 15: 225. 1939.

Adiantum poiretii Wiktröm var. sulphureum (Kaulf.) R. Tryon, Amer. Fern. Jour. 47: 139. 1957.

Adiantum chilense (Kaulf.) var. sulphureum (Hook.) Giucide, Darwiniana 37(3-4): 287. 1999.

Materiales estudiados Seleccionados: Región de O’Higgins: Talcarehue. San Fernando. 30-XII-1950. M. Ricardi s/n (CONC); Rancagua, Cajón de los Cipreses. I-1985. F. Silva s/n (SGO). Región del Maule: Reserva Los Ruiles de Empedrado,
300 m. 9-I-1999. O. Matthei 897 (CONC); Faldas del Cerro Gupo, Dpto. Loncomilla. 4-IX-1939. M.R. Espinosa s/n (SGO). Región del Biobío: Antuco, $11 \mathrm{Km}$ más abajo del Refugio de la Universidad de Concepción. 3-XII-1987. I. Meza y E. Barrera 1717 (SGO); Prov. Concepción. Parque Hualpén, 60 m. 7-I-1976. C. Marticorena, R. Rodríguez y E. Ugarte 1825 (CONC). Región de La Araucanía: Capitán Pastene, 400 m. 8-XII-1934. G. Montero 2246 (CONC); Río Toltén, bosque cerca de Pitrufquén +/- 100 m s.n.m. 9-XII1947. B. Sparre 3449 (SGO). Región de Los Ríos: Llifén. Lago Ranco. 14-I-1942. A. Pfister s/n (CONC); Mancera. 12-II-1969. E. Navas 3380 (SGO). Región de Los Lagos: Parque Nacional V. Pérez Rosales. Petrohué. 210 m. 10-XII1971. C. Marticorena, E. Weldt y J. Crisci 2013 (CONC); Trayecto Petrohué-Saltos del Petrohué. 21-I-1975. E. Barrera y M. Rancusi 9 (SGO). Región de Aisén. Coihaique, Aisén. 9-II-1934. M.R. Espinosa s/n (SGO); Lago General Carrera. Puerto Guadal, 480 m. 31-III-1967. T. Seki 635 (CONC); Prov. Capitán Prat. Valle Grande, El Salto. 26-IV-2006. R. Rodríguez y E. Teneb 4207 (CONC).

Adiantum sulphureum Kaulf. var. majus Hook., Sp. Filic. 2: 44, tab. 76 A, figs. 3, 4. 1851. 
Adiantum sulphureum sensu Remy in Gay, Fl. Chile 6: 486. 1854, non Kaulf. 1824. Typus "Province de Colchagua, au Chili, Gay 1833" (Phototypus P!),

Adiantum sulphureum Kaulf. var. cuneifolium Meigen, Bot. Jahrb. Syst. 17: 218. 1893.

Materiales estudiados Seleccionados: Región de Coquimbo: Depto. Ovalle. Monte Redondo. 17-XI-1947. C. Jiles 489 (CONC); Región de Valparaíso: Cerro La Campana. 11IV-1936. K. Behn s/n (CONC); Olmué. 6-II-1966. M.R. Espinosa s/n (SGO). Región Metropolitana: Quebrada de Peñalolén. 10-XII-1965. M. Mahú 2574 (CONC); Cajón del Maipo, frente desembocadura El Manzano, más o menos 3 km al N. 880 m s.n.m. 30-XI-1994. M. Muñoz y A. Moreira 3411 (SGO); Reserva Nacional Río Clarillo, $3 \mathrm{~km}$ al $\mathrm{N}$ de entrada a la Reserva (3343'47";70²8'9") 963 m. 6-XII2004. M. Bonifacio 1330 (SGO). Región de O’Higgins: La Rufina. Río Claro. 3-I- 1951. M. Ricardi s/n (CONC); Termas de Cauquenes. 3-XI-1952. A. Pfister s/n (CONC); Cahuil. Prov. Colchagua. Aprili 1831. C. Gay s/n (SGO). Región del Maule: Linares. 12-II- 1947. O. Barros s/n (CONC); Los Queñes, cruzando el Río Teno. 09-III-1996. J. Arriagada 79 (SGO). Región del Biobío: Concepción, cerros de La Toma. 29-XII-1934. C. Junge s/n (CONC); Penco. 16-X-1923. H. Gunckel 2504 (CONC). Región de La Araucanía: Cautín, Almagro. 21-II-1943. H. Gunckel 14185 (CONC).

\section{Asplenium fragile C. Presl var. lomense Weath.}

De acuerdo a Tryon \& Stolze (1993) Asplenium fragile es un sinónimo de Asplenium peruvianum Desv. y por lo tanto incluye la variedad lomense de Weatherby publicada en Johnston (1929), entonces en la flora de Chile se debe citar a A. peruvianum con la única localidad mencionada por Johnston (1929).

Asplenium peruvianum Desv., Mém. Soc. Linn. Paris 6: 271. 1827.
Sinónimo para las plantas de Chile: Asplenium fragile C. Presl var. lomense Weath., in I.M. Johnston, Contr. Gray Herb. 85: 13. 1929. Typus: "Chile; under rocks in bottom of the gulch above the water-hole at Aguada del Panul, Dept. Taltal, 4-XII-1925, Johnston 5421 (Type, GH)"

La única muestra chilena conocida es la que se encuentra en GH recolectada por I.M. Johnston en la Aguada del Panul, cerca de Taltal en 1925, no existen noticias posteriores a este hallazgo y este taxón todavía permanece dudoso para la flora de Chile.

\section{BLECHNUM BLECHNOIDES KEYSERL.}

Blechnum blechnoides Keyserl. es un helecho que se encuentra generalmente muy cerca del nivel del mar y precisamente el tipo proviene del área de Concepción-Talcahuano, donde crece al abrigo de las rocas litorales, incluso a veces salpicado por el agua salina; este ambiente le otorga características propias, como por ejemplo su lámina estéril que es carnosa y con la venación poco notoria. Looser (1947) dice "conocer esta planta únicamente desde Talcahuano ( $\left.36^{\circ} 45^{\prime} \mathrm{S}\right)$, prov. de Concepción hasta Ancud ( $41^{\circ} 50^{\prime} \mathrm{S}$ ) en la isla de Chiloé y siempre cerca del mar'. De acuerdo a lo anterior $B$. blechnoides es endémico de Chile y su estado de conservación es Vulnerable (Baeza et al. 1998).

Chambers \& Farrant (1996) agregan como sinónimo de Blechnum blechnoides a Blechnum banksii (Hook.f.) Mett. ex Diels, de Nueva Zelandia, sin embargo se trata de dos especies con características morfológicas muy diferentes en cuanto a la arquitectura y contorno de las láminas estériles y fértiles, longitud del pecíolo, ápice de las pinnas, entre otros; además se encuentran separadas por una gran extensión de océano sin registros en lugares intermedios.

Por mucho tiempo existió una confusión taxonómica por su parecido entre B. blechnoides y B. mochaenum Kunkel, esta última especie abunda en los bosques del sur de Chile y no tan cerca del litoral marino, y su lámina estéril es herbácea y con nervios notorios. Ambas entidades se pueden separar mediante la siguiente clave (en ejemplares maduros):

1. Hojas de 10-25 cm de largo. Lámina linear-lanceolada, con la venación poco notoria; pecíolo de $2 \mathrm{~cm}$ o casi nulo. Pinnas centrales ligeramente falcadas, aovado-oblongas, glabras, obtusas, con el ápice redondeado, las inferiores se achican gradualmente hacia la base de la lámina....

Blechnum blechnoides

1'. Hojas de 30-40 cm de largo. Lámina lanceolada a oblongo-lanceolada, con la venación notoria; pecíolo de 3-10 cm de largo. Pinnas centrales alargadas y falcadas acroscópicamente, con algunas escamas finas en la cara inferior, con el ápice generalmente agudo, las inferiores semicirculares aumentando en largo gradualmente hacia el centro.

Blechnum mochaenum

Blechnum blechnoides Keyserl., Polypodiaceae et Cyatheaceae herbarii Bungeani 65. 1873. "Chile, Concepcion, leg. Dumont d'Urville, 1824".

Sinónimos: Lomaria leyboldtiana Phil., Anales Univ. Chile 18: 68. 1861; Blechnum leyboldtianum (Phil.) C. Chr., Index Filic. 156. 1905.

Materiales estudiados seleccionados: Región del Maule: Prov. de Cauquenes. Sector de Tregualemu. 17-X-2010.
P. Bravo y G. Baeza s/n (CONC). Región del Biobío: Laraquete, rocas de la playa. 20-XII-1949. M. Ricardi s/n (CONC); Talcahuano. Rocoto. 20-IV-1970. E. de la Sota y R. Rodríguez s/n (CONC). Región de La Araucanía: Puerto Saavedra. 30-XII-1932. G. Montero 1142 (CONC); Boca Budi. II-1925. M.R. Espinosa s/n (SGO). Región de Los Ríos: Valdivia, Corral, Gruta La Aguada. 3-VIII-1939. H. Gunckel 16253 (CONC); Valdivia. Hueicolla. 10-II-1988. M.F. Gardner \& S.G. Knees 4148 (CONC); La Barra del Río Bueno, rocas del mar. 18-II-1948. B. Sparre 4547 (SGO). 
Región de Los Lagos: Osorno, Pucatrihue. 28-XII-1953. B. Sparre \& Smith 298 (CONC); Ancud. Fuerte San Antonio. 12-IV-1938. G. Looser 3849 (CONC); Chiloé continental. Huinay. 7-I-1981. B. Parra s/n (CONC).

Blechnum chilense (Kaulf.) Mett.

Blechnum chilense (Kaulf.) Mett. es el nombre correcto para el helecho chileno que se caracteriza por el dimorfismo foliar, con frondes estériles que pueden alcanzar los 150 $\mathrm{cm}$ de largo, a veces los rizomas y pecíolos viejos forman un pequeño tronco de $50 \mathrm{~cm}$ de alto por $15 \mathrm{~cm}$ de diámetro, escamas del pecíolo abundantes, de color castaño claro, basifijas, las pinnas son coriáceas, cortamente pecioluladas y con el margen ondulado. Las esporas fueron estudiadas por Rodríguez (1970) y allí señala que son plano-convexas de 4455 x 60-70 x 51-57 micrones, margen de la laesura grueso, ondulado y con pequeñas perforaciones.

Tryon \& Stolze (1993) incluyeron a Blechnum chilense bajo la sinonimia de B. cordatum (Desv.) Hieron. y este mismo criterio adoptan Prada et al. (2008) dando una gran distribución pantropical que incluye Mesoamérica, Antillas, Sudamérica, Archipiélago de Juan Fernández, Hawai y SE de Australia. En un trabajo anterior Rolleri \& Prada (2006) consideran a $B$. chilense como integrante de las floras de Ecuador, Brasil meridional (dudoso), Chile, Uruguay y Argentina. El grupo de especies sudamericanas de hábito cicadiforme son de aspecto similar, sin embargo, las diferencias morfológicas detalladas entre $B$. chilense y $B$. cordatum, como por ejemplo la forma y distribución de las escamas del pecíolo y raquis, los aeróforos y la palinología, permiten separar claramente los dos taxones.

De la Sota (1972) considera que B. chilense es un elemento austral-antártico, vicariante con Blechnum capense L., que vive en Chile continental, Juan Fernández y Argentina, opinión que es compartida en este trabajo.

Blechnum chilense (Kaulf.) Mett., Fil. Lechl. 1: 14. 1856.

Basiónimo: Lomaria chilensis Kaulf., Enum Filic. 154. 1824. Typus. "Habitat in Chile, Chamisso" (región vecina a Talcahuano-Concepción, única parte de Chile donde estuvo Chamisso).

Materiales estudiados Seleccionados: Región de Coquimbo: Depto. Illapel. Naigué. 20-X-1955. C. Jiles 2831-a (CONC); Los Vilos. XI-1956. H. Gunckel 57220 (CONC). Región de Valparaíso: Camino de Viña a Concón. 18-II-1940. K. Behn s/n (CONC). Archipiélago de Juan Fernández: Masatierra, Quebrada de la Damajuana, 400 m. 3-III-1955. B. Sparre 272 (CONC). Región Metropolitana: Santiago. El Canelo (Cajón del Maipo). II-1957. P. Valenzuela s/n (CONC). Región de O’Higgins: Prov. Cardenal Caro. Tanumé. Loma Clavija. 29I-2005. I. Vera y N. Ortiz 177. (CONC). Región del Maule: Reserva Los Ruiles de Empedrado. 9-I-1999. O. Matthei
905 (CONC); Alto de Vilches. Camino a Laguna El Alto. 29-I-2000. V. Finot \& P. López 1728 (CONC). Región del Biobío: Contulmo. 26-XII-1935. G. Montero 2756 (CONC); Concepción, Cerro Caracol. 3-I-1941. H. Gunckel 10161 (CONC). Región de La Araucanía: Cordillera de Nahuelbuta, Camino de Quidico a Relún. 7-I-1977. C. Marticorena, M. Quezada y R. Rodríguez 1197 (CONC); Temuco. Cerro Ñielol. 3-III-1946. H. Gunckel 15570 (CONC). Región de Los Ríos: Lago Riñihue, lado norte. 21-XII-1974. G. Montero 9521 (CONC); Valdivia. Los Guindos. 28-I-1941. H. Gunckel 15317 (CONC). Región de Los Lagos: Prov. Chiloé. Isla Alao. 6-II 1983. I. Meza y G. Valenzuela 5082 (CONC); Ancud. 7-I-1924. E. Barros 962 (CONC). Región de Aisén: Puyuhuapi. 13-IV-1940. H. Schwabe 9 (CONC); Tortel. 5-VIII-1976. R. Rodríguez 809 (CONC). Región de Magallanes: Puerto Natales. Seno Wald. 19-XI-2009. J.C. Aravena, R. Villa, E. Domínguez, J.M. Henríquez, C. Galleguillos y K. Haro 518 (CONC).

Botrychium australe R.BR. SUBSP. NEGERI (Chist) R.T. Clausen

Se han descrito dos subespecies de Botrychium australe R. $\mathrm{Br}$., B. australe subsp. australe que es endémica de Argentina (Neuquén y Río Negro) y B. australe subsp. negeri (Christ) R.T. Clausen que también crece en Argentina y ha sido citado erróneamente para Chile.

La presencia en Chile de Botrychium australe subsp. negeri no ha sido confirmada y De la Sota et al. (1998) dicen que la presencia en Chile es cuestionada. Botrychium negeri Christ, de acuerdo al protólogo de su descripción original "Hab. S. Chile, Valdivia; leg. Dr. Neger 1897" y no ha sido encontrado otra vez por más de cien años en esta región a pesar de haber sido muy bien estudiada bajo el punto de vista florístico. Looser (1938: 264) sospecha que se trate de una planta coleccionada por Neger (1899) en su excursión a la cordillera de Villarrica y que citó bajo el nombre de Botrychium ternatum Sw. Del texto de Neger no se desprende claramente que lo haya encontrado en suelo chileno y el ejemplar depositado en el Herbario del Museo Nacional (SGO) está acompañado por una etiqueta que dice: "Botrychium ternatum, pampa de Huihui, cerca del lago Quillén”. Esta localidad está en Argentina.

\section{REVALIDACIÓN DE EQUISETUM PYRAMIDALE GOLDM.}

Equisetum giganteum L. es la especie que alcanza el mayor tamaño dentro del género Equisetum que se encuentra creciendo en casi todo el continente americano (Looser 1961, Rodríguez 1995, De la Sota 1998), desde Cuba, Jamaica y Haití, sur de México, América Central hasta Chile y Argentina. En nuestro país su presencia se limita solamente a la Región de Tarapacá, pero Rodríguez (l.c.) señala que los individuos de las poblaciones que se encuentran en las provincias centrales de Chile son más pequeños y de menor diámetro y 
Gayana Bot. 72(1), 2015

posiblemente se trata de otra entidad taxonómica, que ahora se propone restablecer como Equisetum pyramidale Goldm.

Efectivamente existen diferencias morfológicas que separan a Equisetum pyramidale de E. giganteum que se expresan en la clave, y además por presentar estróbilos elípticos o algo redondeados, apiculados; esporangióforo hexagonal con un punto negro en el centro, con seis esporangios.

Ambas especies se pueden separar mediante la siguiente clave:

1. Tallos de 0,7 a 1,5 cm de diámetro, débiles, con costillas definidas algo sobresalientes; vainas del tallo principal con 6-9 dientes

Equisetum pyramidale

1'. Tallos mayores de 1,5 cm de diámetro, rígidos, costillas no sobresalientes; vainas del tallo principal con más de 10 dientes..

Equisetum giganteum

Equisetum pyramidale Goldm., Nov. Actorum Acad. Caes. Leop.-Carol. Nat. Cur. 11: 469. 1843. Typus: "Chile: Melipilla". Equisetum scandens J. Remy in Gay, Hist. Chile, Bot. 6: 471. 1854. Typus: "Especie de los lugares cenagosos de Quillota y notable por sus tallos que suben a los árboles".

Equisetum araucanum Phil., Anales Univ. Chile 94: 352. 1896. Typus: "Habitat in Araucania et quidem praedio Renaico, ubi rarum videtur". (SGO!)

Equisetum giganteum L. var. chilense Milde, Ver. K.K. Zool.Bot. Ges. Wien 13: 229.1863. Typus: "C. Gay herb. Fée".

Equisetum ramosissimum Desf. var. scaberium Milde, Monogr. Equiset. 443. 1867. Typus: "Prope urbem Valparaiso in republica Chilensi, ubi lectum est a Jelinek in expeditiones Novarae (Herb. Caesar. Vondob.)".

Equisetum ramosissimum Desf. var. affine Milde, Monogr. Equiset. 453. 1867. Typus: "Santiago de Chile".

Equisetum philippii Gand., Bull. Soc. Bot. France 66: 304. 1920. Typus: "Chili (Philippi!)".

De acuerdo a los materiales revisados se encuentra en Chile desde la provincia de Limarí hasta la provincia de Malleco. Es una planta marcadamente higrófila, crece en lechos de río, a lo largo de los canales, en fosos, vegas húmedas, incluso en terrenos agrícolas o alterados.

Materiales estudiados Seleccionados: Región de Coquimbo: Huentelauquén. Río Choapa. III-1957. G. Monsalve s/n (SGO); Paihuano. 18-18-X-1948. A. Pfister (CONC); Vallenar, Embalse Santa Juana (28 34’; 7045’) 585 m. F. Saavedra 491 (SGO). Región de Valparaíso: Papudo. VII1960. M.L. Véliz s/n (SGO); Depto. Quillota. Estación La Cruz. 29-IV-1928. M. Espinosa s/n (SGO); Prov. Quillota. Hijuelas. 12-XI-1987. O. Matthei y M. Quezada s/n (CONC); Limache. 28-XI-1927. G. Looser s/n (CONC). Región Metropolitana: Peñalolén, Quebrada El Abanico. 24-61916. P.N. Cortés s/n (SGO); Cajón del Maipo, Río Yeso. 4-I-1990. Ch. von Bohlen 709 (SGO); Cerca de Paine, a orillas Carretera Panamericana. 24-XI-1968. M. Mahú 4068 (CONC); Las Vertientes, en algunos matorrales cerca del río Maipo. 29-IX-1951. H. Gunckel 24600 (CONC). Región de O'Higgins: Requinoa, a orillas de un canal de riego. XII1959. H. Gunckel 34687 (CONC). Región del Maule: San Javier de Loncomilla. VIII-1918. s/col. (SGO). Región del
Biobío: Concepción, Perales, orilla línea férrea a Talcahuano. 27-III-1934. M. Espinosa s/n (SGO); Concepción, en una quebrada del Cerro Caracol. 8-XII-1923. H. Gunckel 187 (CONC); Talcahuano, humedales al lado de Carriel Sur. 25-II-1994. A. Marticorena 68 (CONC); Quillón. Fundo El Roble. 27-III-1998. L. Finot 490 (CONC). Región de La Araucanía: Camino Renaico, Estación Roblería (orillas del terraplén del FFCC). II-1951. Pfister s/n (CONC). Renaico, crece a la orilla de la línea férrea, entre zarzamora. 3-XI1965. G. Montero 7316 (CONC).

Polypodium masafuerae y su presencia en el norte De Chile

Polypodium masafuerae Phil., Linnaea 29: 107. 1858. Tipo: "In insula Masafuera a Germainio lectum est" (SGO!).

Pleopeltis masafuerae (Phil.) de la Sota, Darwiniana 45: 239. 2007. Polypodium macrocarpum sensu Hooker, Century Ferns, tab. 34. 1854, non Presl.

Plantas de 7-17 cm de alto, escamas del rizoma filiformes. Lámina de 4-10,5 cm de largo, pecíolo glabro o con pocas escamas basifijas, raquis escamoso. Pinnas con muy pocas escamas, como las del pecíolo pero más pequeñas; pinnas basales de 1-3 cm de largo con el margen sinuado-crenado.

Se conocen pocos hallazgos de este helecho de la isla Alejandro Selkirk del Archipiélago de Juan Fernández, el primero de ellos fue descrito e ilustrado por Hooker (1854, tab. 34) bajo el nombre de Polypodium macrocarpum Presl sobre la base del material de "Massa [sic] Fuera, S. Pacific, lat $34^{\circ}$, Cuming, n. 1352"); otra muestra fue obtenida por Philibert Germain, descrita por R.A. Philippi (1858) como Polypodium masafuerae y finalmente C. Muñoz y E. Sierra recolectaron material en la Quebrada de la Colonia en 1965.

Rodríguez (1995) indica erróneamente que Polypodium masafuerae crece en la provincia de Antofagasta, además se encuentra en el sur de Perú y en la Isla Alejandro Selkirk del Archipiélago de Juan Fernández, afirmación que se viene repitiendo desde que Hooker (1854) coloca en los materiales estudiados al ejemplar de H. Cuming entre otros, de Perú y Bolivia, bajo el nombre de Polypodium macrocarpum C.Presl, que es un nombre ilegítimo y ahora se considera como sinónimo de Polypodium pycnocarpum. 
En consecuencia, Polypodium masafuerae es una buena especie y es endémica de la Isla Alejandro Selkirk del Archipiélago de Juan Fernández. Estado de conservación En Peligro.

Materiales estudiados: Región de Valparaíso, Provincia de Valparaíso, Archipiélago de Juan Fernández: Masafuera. Germain s/n (Typus de Polypodium masafuerae SGO 083268); Isla de Más a Fuera, Quebrada de La Colonia, entre el último Eucalyptus y Morro Capitán. 27-XI-1965, alt. 200 m s.n.m. C. Muñoz y E. Sierra 7009 (SGO).

Las referencias bajo el nombre de Polypodium masafuerae citadas para el norte de Chile (Hooker 1854, Looser 1952, Rodríguez 1995) se deben cambiar a Polypodium pycnocarpum y de acuerdo a Tryon \& Stolze (1993) se encuentra en Perú, Bolivia, Chile y Argentina. En el país solamente se ha recolectado en la costa de Paposo, provincia de Antofagasta, y estas poblaciones se consideran en estado de conservación En Peligro.

Polypodium pycnocarpum C. Chr., Index Filicum 326. 1905. 557. 1906.

Sinónimos (para la flora de Chile): Polypodium macrocarpum Presl, Rel. Haenk. 1: 23. 1825, non Bory ex Willd. 1810; Polypodium molledense Maxon, Smithsonian Misc. Collect. 65(8): 1. 1915; Polypodium squamatum Phil., Linnaea 29: 107. 1857 (nomen), Fl. Atacam. 56. 1860. Typus "Prope Paposo in regione herbifera 500-1000 p. s. m. inveni” (SGO!); Polypodium atacamense Baker ex Ball, J. Linn. Soc., Bot. 22: 64. 1885. [P. atacamense es un simple cambio de nombre en lugar de $P$. squamatum Phil. por haber sido usado este binomio ya por Linneo en 1753].

Materiales estudiados: Región de Antofagasta: Paposo. N 133. [tipo de Polypodium squamatum Phil., plantas de 5-6 cm] (SGO); Paposo, in des. Atac. [isotipo de Polypodium squamatum Phil.), frondes de $5 \mathrm{~cm}$. El ejemplar está acompañado de 3 etiquetas: a) Herb. H. Christ, Bale. Polypodium macrocarpum Presl, Rel. Haenk. 1. 23. Tab. 1. 4; b) Polyp. pycnocarpum C. Chr. (Polypodium atacamense Bak.), letra desconocida; c) Polypodium squamatum Ph., Paposo in des. Atac. [letra de Philippi] (SGO); Vicinity of Aguada de Miguel Díaz, ca. 24³5' S. 1-4-XII-1925. Growing in moss in crotch of an arborescent Cereus. I.M. Johnston 5309 (GH); Dept. Taltal. Aguada del Panul, ca. lat. $24^{\circ} 47^{\prime}$ S. 4-XII-1925. In moss on face of rock in quebrada above water hole. I.M. Johnston 5423 (GH); Dept. Taltal. El Rincón, just north of Paposo, along trail to old Parañas Mine, ca. lat. 24'59' S. 7-XII-1925. I.M. Johnston 5491 (GH, SGO); El Rincón, al N. de Paposo; suelo arenillo arenoso. 17IX-1941. C. Muñoz \& G.T. Johnson 2921 (SGO); Quebrada El Médano, al N de Paposo; suelo arenoso. En la grieta de las rocas, alt. ca. 460 m s.n.m. 8-X-1941. E. Pisano \& R. Bravo
389 (SGO); Rinconada de Paposo, Quebrada San Miguel. 350 m s.m. (245' S -70²8' W). 4-XII-1988. A. Hoffmann 300 (CONC); Quebrada Rinconada, ca. $5 \mathrm{~km} \mathrm{~N}$ of Caleta Paposo, ca. $550 \mathrm{~m}$ alt. $\left(24^{\circ} 56^{\prime} \mathrm{S} ; 70^{\circ} 30^{\prime} \mathrm{W}\right)$. Terrestrial, growing in protected rocky crevices. 19-XI-1988. M.O. Dillon, D. Dillon, V. Asencio \& M. Villarroel 5993 (CONC, SGO).

\section{AGRADECIMIENTOS}

Al Proyecto de la Nueva Flora de Chile y al personal del Herbario CONC por el apoyo prestado en todo momento, a Gloria Rojas por las facilidades otorgadas para la revisión de materiales del Herbario SGO y a Mélica Muñoz por el envío de fotografías del tipo de Polypodium masafuerae.

\section{BIBLIOGRAFÍA}

Baeza, M., E. Barrera, J. Flores, C. Ramírez \& R. Rodríguez. 1998. Categorías de conservación de Pteridophyta nativas de Chile. Boletín del Museo Nacional de Historia Natural 47: 23-46.

Chambers, T.C. \& P.A. FArRant. 1996. Blechnum blechnoides (Bory) Keys. (Blechnaceae), formely B. banksii (Hook.f.) Mett. ex Diels, a fern from salt-spray habitats of New Zealand and Chile. New Zealand Journal of Botany 34: 441-445.

De LA Sota, E.R. 1972. Notas sobre especies austrosudamericanas del género Blechnum L. (Blechnaceae-Pteridophyta). III. Boletín de la Sociedad Argentina de Botánica 14(3): 177184.

De La Sota, E.R. 1998. Pteridophyta. En: M.N. Correa (ed.), Flora Patagónica Parte I. Colección Científica INTA: 282-369.

Giudice, G.E. 1999. Sinopsis de las especies argentinas del género Adiantum (Pteridaceae, Pteridophyta). Darwiniana 37(3-4): 279-300.

Holmgren, P.K., N.H. Holmgren \& L.C. Barnett. 1990. Index Herbariorum: The herbaria of the world. Volumen 120 de Regnum vegetabile. Ed. International Association for Plant Taxonomy by New York Botanical Gardens. 693 pp.

HOOKER, W.J. 1854. A century of ferns; being figures with brief descriptions of one hundred new, or rare, or imperfectly known species of ferns, from various parts of the world; a selection from author's "Icones plantarum," ... London. vii pp., 100 plates.

HoOKer, W.J. 1858. Species filicum. London. Vol. 2. 250 pp., 140 lám. Johnston, I.M. 1929. Papers on the flora of northern Chile. 1. The coastal flora of the departments of Chañaral and Taltal; 2. The flora of the Nitrate Coast; 3. Undescribed species from the cordilleras of Atacama. Contributions from the Gray Herbarium 85: 1-172.

KAULFUSS, G.F. 1824. Enumeratio filicum quas in itinere circa terram legit C. Adalbertus de Chamisso. Leipzig. vi, 300 pp., 2 lám.

Looser, G. 1938. Catálogo de las plantas vasculares nuevas de Chile. Revista Universitaria, Universidad Católica de Chile 23(2): 215-275.

Looser, G. 1947. Los Blechnum (Filices) de Chile. Revista Universitaria, Universidad Católica de Chile 32(2): 9-106. 
Looser, G. 1952. El género Polypodium L. y sus representantes chilenos. Revista Universitaria, Universidad Católica de Chile 36(1): 13-82, 17 figs.

Looser, G. 1961. Los Pteridófitos o helechos de Chile (excepto Isla de Pascua) I. Revista Universitaria, Universidad Católica de Chile 46: 213-262, 17 lám.

Meigen, F. 1893. Skizze der Vegetationsverhältnisse von Santiago in Chile. Botanische Jahrbücher für Systematik, Pflanzengeschichte und Pflanzengeographie 17: 199-294.

Neger, F.W. 1899. Informe sobre las observaciones botánicas efectuadas en la cordillera de Villarrica en el verano 189697. Anales de la Universidad de Chile 103: 903-967.

PHILIPPI, R.A. 1858. Plantarum novarum chilensium. Centuria sextae pars. Linnaea 29: 96-110.

Prada, C., C. Rolleri \& L.Passarelli. 2008. Morfología, caracterización y distribución geográfica de Blechnum cordatum (Blechnaceae-
Pteridophyta). Acta Botanica Malacitana 33: 29-46.

Remy, J. 1854. Helechos. En C. Gay, Historia Física y Política de Chile, Botánica. Vol 6: 473-542.

RodRíGuez, R. 1970. Morfología de las esporas, protalos y esporofitos jóvenes de algunas especies chilenas del género Blechnum L. (Polypodiaceae). Tesis Lic. Biología. Universidad de Concepción. Chile. 91 pp.

Rodríguez, R. 1995. Pteridophyta. En: C. Marticorena y R. Rodríguez (eds.), Flora de Chile 1: 119-309.

Rolleri, C. \& C. Prada. 2006. Catálogo comentado de las especies de Blechnum L. (Blechnaceae, Pteridophyta) de Mesoamérica y Sudamérica. Anales del Jardín Botánico de Madrid 63(1): 67-106.

Tryon, R.M. \& R.G. Stolze. 1993. Pteridophyta of Peru. Part V. 18. Aspleniaceae - 21. Polypodiaceae. Fieldiana Botany (n.s.) 32: 1-190.

Recibido: 13.01.14

Aceptado: 12.11.14 